\title{
New symmetric designs from regular Hadamard matrices
}

\author{
Yury J. Ionin* \\ Department of Mathematics \\ Central Michigan University \\ Mt. Pleasant, MI 48859, USA \\ yury.ionin@cmich.edu
}

November 29, 1997

\begin{abstract}
For every positive integer $m$, we construct a symmetric $(v, k, \lambda)$-design with parameters $v=\frac{h\left((2 h-1)^{2 m}-1\right)}{h-1}, k=h(2 h-1)^{2 m-1}$, and $\lambda=h(h-1)(2 h-1)^{2 m-2}$, where $h= \pm 3 \cdot 2^{d}$ and $|2 h-1|$ is a prime power. For $m \geq 2$ and $d \geq 1$, these parameter values were previously undecided. The tools used in the construction are balanced generalized weighing matrices and regular Hadamard matrices of order $9 \cdot 4^{d}$.
\end{abstract}

Submitted: October 30, 1997; Accepted: November 17, 1997

MR Subject Number: 05B05

Keywords: Symmetric design, regular Hadamard matrix, balanced generalized weighing matrix

\section{Introduction}

Let $v>k>\lambda \geq 0$ be integers. A symmetric $(v, k, \lambda)$-design is an incidence structure $(P, \mathcal{B})$, where $P$ is a set of cardinality $v$ (the point-set) and $\mathcal{B}$ is a family of $v k$-subsets (blocks) of $P$ such that any two distinct points are contained in exactly $\lambda$ blocks. If $P=\left\{p_{1}, \ldots, p_{v}\right\}$ and $\mathcal{B}=\left\{B_{1}, \ldots, B_{v}\right\}$, then the $(0,1)$-matrix $M=\left[m_{i j}\right]$ of order $v$, where $m_{i j}=1$ if and only if $p_{j} \in B_{i}$, is the incidence matrix of the design. A $(0,1)$-matrix $X$ of order $v$ is the incidence matrix of a symmetric $(v, k, \lambda)$-design if and only if it satisfies the equation $X X^{T}=(k-\lambda) I+\lambda J$, where $I$ is the identity matrix and $J$ is the all-one matrix of order $v$. For references, see [1] or [3, Chapter $5]$.

A Hadamard matrix of order $n$ is an $n$ by $n$ matrix $H$ with entries equal to \pm 1 satisfying $H H^{T}=n I$. A Hadamard matrix is regular if its row and column sums are constant. This sum is always even and if we denote it $2 h$, then the order of the matrix is equal to $4 h^{2}$. Replacing $-1 \mathrm{~s}$ in a regular Hadamard matrix of order $4 h^{2}$ by 0 s yields the incidence matrix of a symmetric $\left(4 h^{2}, 2 h^{2}-h, h^{2}-h\right)$-design usually

\footnotetext{
${ }^{*}$ The author acknowledges with thanks the Central Michigan University Research Professor award.
} 
called a Menon design. Conversely, replacing 0s by $-1 \mathrm{~s}$ in the incidence matrix of a symmetric $\left(4 h^{2}, 2 h^{2}-h, h^{2}-h\right)$-design yields a regular Hadamard matrix of order $4 h^{2}$. For references, see [9]. In this paper, we will be interested in regular Hadamard matrices of order $9 \cdot 4^{d}$, where $d$ is a positive integer. If $H$ is such a matrix, then the Kronecker product of a regular Hadamard matrix of order 4 and $H$ is a regular Hadamard matrix of order $9 \cdot 4^{d+1}$. Therefore, one can obtain a family of regular Hadamard matrices of order $9 \cdot 4^{d}$, starting with a regular Hadamard matrix of order 36 .

A balanced generalized weighing matrix $\mathrm{BGW}(v, k, \lambda)$ over a (multiplicatively written) group $G$ is a matrix $W=\left[\omega_{i j}\right]$ of order $v$ with entries from the set $G \cup\{0\}$ such that (i) each row and each column of $W$ contain exactly $k$ non-zero entries and (ii) for any distinct rows $i$ and $h$, the multiset

$$
\left\{\omega_{h j}^{-1} \omega_{i j}: 1 \leq j \leq v, \omega_{i j} \neq 0, \omega_{h j} \neq 0\right\}
$$

contains exactly $\lambda /|G|$ copies of every element of $G$.

In this paper, we will use a balanced generalized weighing matrix $\operatorname{BGW}\left(q^{m}+\right.$ $q^{m-1}+\cdots+q+1, q^{m}, q^{m}-q^{m-1}$ ) over a cyclic group $G$ of order $t$, where $q$ is a prime power, $m$ is a positive integer, and $t$ is a divisor of $q-1$. Such matrices are known to exist [3, IV.4.22] and have been applied to constructing symmetric designs by Rajkundlia [8], Brouwer [2], Fanning [4], and the author [5, 6]. If $\mathcal{M}$ is a set of $m$ by $n$ matrices, $G$ is a group of bijections $\mathcal{M} \rightarrow \mathcal{M}$, and $W$ is a balanced generalized weighing matrix over $G$, then, for any $P \in \mathcal{M}, W \otimes P$ denotes the matrix obtained by replacing every entry $\sigma$ in $W$ by the matrix $\sigma P$. In Section 2 (Lemma 2.1), we will prove the following modification of a result from [6]:

Let $\mathcal{M}$ be a set of matrices of order $v$ containing the incidence matrix $M$ of a symmetric $(v, k, \lambda)$-design with $q=\frac{k^{2}}{k-\lambda}$ a prime power. Let $G$ be a finite cyclic group of bijections $\mathcal{M} \rightarrow \mathcal{M}$ such that $(\mathrm{i})(\sigma P)(\sigma Q)^{T}=P Q^{T}$ for any $P, Q \in \mathcal{M}$ and $\sigma \in G$, (ii) $\sum_{\sigma \in G} \sigma M=\frac{k|G|}{v} J$, and (iii) $|G|$ divides $q-1$. If $W$ is a balanced generalized weighing matrix BGW $\left(q^{m}+\cdots+q+1, q^{m}, q^{m}-q^{m-1}\right)$ over $G$, then $W \otimes M$ is the incidence matrix of a symmetric $\left(v\left(q^{m}+q^{m-1}+\cdots+q+1\right), k q^{m}, \lambda q^{m}\right)$-design.

In order to apply this lemma, we need a symmetric $(v, k, \lambda)$-design to start with. In the paper [6], we have shown that the designs corresponding to certain McFarland and Spence difference sets (or their complements) serve as such starters. In Section 3 of this paper, we show that for $h= \pm 3 \cdot 2^{d}$, if $|2 h-1|$ is a prime power, then there is a symmetric $\left(4 h^{2}, 2 h^{2}-h, h^{2}-h\right)$-design, which can also serve as a starter. As a result, we show that for any positive integers $m$ and $d$, if $h= \pm 3 \cdot 2^{d}$ and $|2 h-1|$ is a prime power, then there exists a symmetric $(v, k, \lambda)$-design with

$$
v=\frac{h\left((2 h-1)^{2 m}-1\right)}{h-1}, k=h(2 h-1)^{2 m-1}, \lambda=h(h-1)(2 h-1)^{2 m-2} .
$$

These parameters are new, except $m=1$ (Menon designs) and $d=0$ (constructed by the author in $[6])$. 


\section{Preliminaries}

Throughout this paper, we will denote identity, zero, and all-one matrices of suitable orders by $I, O$, and $J$, respectively.

If $W$ is a balanced generalized weighing matrix of order $w$ over a group $G$ of bijections on a set $\mathcal{M}$ of matrices of order $n$, then, for any $P \in \mathcal{M}$, we will denote by $W \otimes P$ the matrix of order $n w$ obtained by replacing every nonzero entry $\sigma$ in $W$ by the matrix $\sigma P$ and every zero entry in $W$ by the zero matrix of order $n$.

The following lemma represents a slight modification of a result proven in [6]. Since it is crucial for this paper and the proof is short, we will repeat it here.

Lemma 2.1 Let $v>k>\lambda \geq 0$ be integers. Let $\mathcal{M}$ be a set of matrices of order $v$ and $G$ a finite group of bijections $\mathcal{M} \rightarrow \mathcal{M}$ satisfying the following conditions:

(i) $\mathcal{M}$ contains the incidence matrix $M$ of a symmetric $(v, k, \lambda)$-design;

(ii) for any $P, Q \in \mathcal{M}$ and $\sigma \in G$,

$$
(\sigma P)(\sigma Q)^{T}=P Q^{T}
$$

(iii) $\sum_{\sigma \in G} \sigma M=\frac{k|G|}{v} J$;

(iv) $q=\frac{k^{2}}{k-\lambda}$ is a prime power;

(v) $G$ is cyclic and $|G|$ divides $q-1$.

Then, for any positive integer $m$, there exists a symmetric $\left(v w, k q^{m}, \lambda q^{m}\right)$-design, where $w=\frac{q^{m+1}-1}{q-1}$.

Proof. Let $W=\left[\omega_{i j}\right], i, j=1,2, \ldots, w$ be a balanced generalized weighing matrix $\operatorname{BGW}\left(w, q^{m}, q^{m}-q^{m-1}\right)$ over $G$. We claim that $W \otimes M$ is the incidence matrix of a symmetric $\left(v w, k q^{m}, \lambda q^{m}\right)$-design. It suffices to show that, for $i, h=1,2, \ldots, w$,

$$
\sum_{j=1}^{w}\left(\omega_{i j} M\right)\left(\omega_{h j} M\right)^{T}=\left\{\begin{array}{l}
(k-\lambda) q^{m} I+\lambda q^{m} J \text { if } i=h, \\
\lambda q^{m} J \text { if } i \neq h .
\end{array}\right.
$$

If $i=h$, we have for some $\sigma_{j} \in G$,

$$
\sum_{j=1}^{w}\left(\omega_{i j} M\right)\left(\omega_{h j} M\right)^{T}=\sum_{j=1}^{q^{m}}\left(\sigma_{j} M\right)\left(\sigma_{j} M\right)^{T}=\sum_{j=1}^{q^{m}} M M^{T}=(k-\lambda) q^{m} I+\lambda q^{m} J .
$$

If $i \neq h$, we have for some $\sigma_{j}, \tau_{j} \in G$,

$$
\begin{gathered}
\sum_{j=1}^{w}\left(\omega_{i j} M\right)\left(\omega_{h j} M\right)^{T}=\sum_{j=1}^{q^{m}-q^{m-1}}\left(\sigma_{j} M\right)\left(\tau_{j} M\right)^{T}=\sum_{j=1}^{q^{m}-q^{m-1}}\left(\tau_{j}^{-1} \sigma_{j} M\right) M^{T} \\
=\frac{q^{m}-q^{m-1}}{|G|}\left(\sum_{\sigma \in G} \sigma M\right) M^{T}=\frac{k\left(q^{m}-q^{m-1}\right)}{v} J M^{T}=\frac{k^{2}\left(q^{m}-q^{m-1}\right)}{v} J=\lambda q^{m} J .
\end{gathered}
$$


Definition 2.2 Let $v>k>\lambda>0$ be integers. $A(v, k, \lambda)$-difference set is a $k$-subset of an (additively written) group $\Gamma$ of order $v$ such that the multiset $\{x-y: x, y \in \Gamma\}$ contains exactly $\lambda$ copies of each nonzero element of $\Gamma$.

Several infinite families of difference sets are known (see [3] or [7] for references). We will mention the McFarland family having parameters $\left(p^{d+1}(r+1), p^{d} r, p^{d-1}(r-1)\right)$, where $p$ is a prime power, $d$ is a positive integer, and $r=\frac{p^{d+1}-1}{p-1}$, and the Spence family having parameters $\left(3^{d+1}\left(3^{d+1}-1\right) / 2,3^{d}\left(3^{d+1}+1\right) / 2,3^{d}\left(3^{d}+1\right) / 2\right)$, where $d$ is a positive integer.

If $\Delta$ is a $(v, k, \lambda)$-difference set in a group $\Gamma$ and $\mathcal{B}=\{\Delta+x: x \in \Gamma\}$, then $\operatorname{dev}(\Delta)=(\Gamma, \mathcal{B})$ is a symmetric $(v, k, \lambda)$-design.

In order to apply Lemma 2.1 , we need a symmetric $(v, k, \lambda)$-design with $q=\frac{k^{2}}{k-\lambda}$ a prime power, a set $\mathcal{M}$ of matrices of order $v$ containing the incidence matrix this design, and a cyclic group $G$ satisfying conditions (ii), (iii), and (v) of Lemma 2.1. In the paper [6], we have shown that $(v, k, \lambda)$ can be the parameters of any McFarland or Spence difference set or their complement with $q=\frac{k^{2}}{k-\lambda}$ a prime power. In this paper, we will use the Spence $(36,15,6)$-difference set in $\Gamma=\mathbb{Z}_{3} \oplus \mathbb{Z}_{3} \oplus \mathbb{Z}_{4}$ and the complementary $(36,21,12)$-difference set. In the next section, we will reproduce the construction of the corresponding $\mathcal{M}$ and $G$ given in [6]

\section{3 (36, 15,6)- and $(36,21,12)$-difference sets}

We start with a brief description of the Spence $(36,15,6)$-difference set in $\Gamma=\mathbb{Z}_{3} \oplus$ $\mathbb{Z}_{3} \oplus \mathbb{Z}_{4}$.

We consider $\Gamma$ as the set of triples $\left(x_{1}, x_{2}, x_{3}\right)$, where $x_{1}, x_{2} \in\{0,1,2\}$ and $x_{3} \in$ $\{0,1,2,3\}$ with the mod 3 and the mod 4 addition, respectively. Consider $\mathbb{Z}_{3} \oplus \mathbb{Z}_{3}$ as a 2-dimensional vector space over the field $\mathrm{GF}(3)$. Let $L_{1}, L_{2}, L_{3}, L_{4}$ be its 1 dimensional subspaces. Put $D_{1}=\left\{\left(x_{1}, x_{2}, 0\right) \in \Gamma:\left(x_{1}, x_{2}\right) \notin L_{1}\right\}$ and, for $i=2,3,4$, $D_{i}=\left\{\left(x_{1}, x_{2}, i-1\right) \in \Gamma:\left(x_{1}, x_{2}\right) \in L_{i}\right\}$. Then $D=D_{1} \cup D_{2} \cup D_{3} \cup D_{4}$ is a $(36,15,6)$ difference set in $\Gamma[7$, Theorem 11.2].

In order to obtain the incidence matrix of the corresponding symmetric design, we have to select an order on $\Gamma$. We will assume that $\left(x_{1}, x_{2}, x_{3}\right)$ precedes $\left(y_{1}, y_{2}, y_{3}\right)$ in $\Gamma$ if and only if there is $i$ such that $x_{i}<y_{i}$ and $x_{j}=y_{j}$ whenever $j>i$. Let $M$ be the $(0,1)$-matrix of order 36 whose rows and columns are indexed by elements of $\Gamma$ in this order and $(x, y)$-entry is equal to 1 if and only if $y-x \in D$. Then $M$ is the incidence matrix of a symmetric $(36,15,6)$-design. In order to describe the structural properties of $M$ which will be important in the sequel, we introduce the following operation $\rho$ on the set of 3 by 3 block-matrices.

Definition 3.1 Let $P=\left[P_{i j}\right]$ be a 3 by 3 block-matrix with square blocks (in particular, $P$ can be a 3 by 3 matrix). Denote by $\rho P$ the matrix obtained by applying the 
cyclic permutation $\rho=(123)$ of degree 3 to the set of columns of $P$, i.e.,

$$
\rho\left[\begin{array}{lll}
P_{11} & P_{12} & P_{13} \\
P_{21} & P_{22} & P_{23} \\
P_{31} & P_{32} & P_{33}
\end{array}\right]=\left[\begin{array}{lll}
P_{13} & P_{11} & P_{12} \\
P_{23} & P_{21} & P_{22} \\
P_{33} & P_{31} & P_{32}
\end{array}\right] .
$$

The above incidence matrix $M$ of a symmetric $(36,15,6)$-design can be represented as a 4 by 4 block-matrix

$$
M=\left[\begin{array}{llll}
M_{1} & M_{2} & M_{3} & M_{4} \\
M_{4} & M_{1} & M_{2} & M_{3} \\
M_{3} & M_{4} & M_{1} & M_{2} \\
M_{2} & M_{3} & M_{4} & M_{1}
\end{array}\right],
$$

where each $M_{i}$ is a 9 by 9 matrix. Further, each $M_{i}$ can be represented as a 3 by 3 block-matrix

$$
M_{i}=\left[\begin{array}{lll}
M_{i 1} & M_{i 2} & M_{i 3} \\
M_{i 3} & M_{i 1} & M_{i 2} \\
M_{i 2} & M_{i 3} & M_{i 1}
\end{array}\right]
$$

where each $M_{i j}$ is a matrix of order $3, M_{11}=O, M_{12}=M_{13}=J, M_{21}=M_{22}=$ $M_{23}=M_{31}=M_{41}=I, M_{32}=M_{43}=\rho I$, and $M_{33}=M_{42}=\rho^{2} I$.

Let $\mathcal{M}$ be the set of block-matrices $P=\left[P_{i j}\right], i, j=1,2,3,4$, where each $P_{i j}$ is a block-matrix $P_{i j}=\left[P_{i j k l}\right], k, l=1,2,3$, satisfying the following conditions:

(i) each $P_{i j k l}$ is a $(0,1)$-matrix of order 3 ;

(ii) for $i=1,2,3,4$, there is a unique $h_{i}=h_{i}(P) \in\{1,2,3,4\}$ such that

$$
\left(P_{i j k 1}, P_{i j k 2}, P_{i j k 3}\right) \text { is a permutation of }(O, J, J) \text { for } j=h_{i} \text { and all } k
$$

and

$$
P_{i j k l} \in\left\{I, \rho I, \rho^{2} I\right\} \text { for } j \neq h_{i} \text { and all } k, l .
$$

Clearly, the above matrix $M$ is an element of $\mathcal{M}$.

Define a bijection $\sigma: \mathcal{M} \rightarrow \mathcal{M}$ by $\sigma P=P^{\prime}$, where

(i) for $i=1,2,3,4$ and $j=2,3,4, P_{i j}^{\prime}=P_{i, j-1}$;

(ii) for $i=1,2,3,4$, if $h_{i}=4$, then $P_{i 1}^{\prime}=\rho P_{i 4}$;

(iii) for $i=1,2,3,4$, if $h_{i} \neq 4$, then $P_{i 1 k l}^{\prime}=\rho P_{i 4 k l}$ for all $k, l$.

Let $G$ be the cyclic group generated by $\sigma$. Then $|G|=12$.

Claim. For any $P, Q \in \mathcal{M},(\sigma P)(\sigma Q)^{T}=P Q^{T}$.

Proof. Let $P, Q \in \mathcal{M}$ and let $P^{\prime}=\sigma P$ and $Q^{\prime}=\sigma Q$. It suffices to show that, for $i=1,2,3,4$,

$$
P_{i 1}^{\prime} Q_{i 1}^{\prime T}=P_{i 4} Q_{i 4}^{T}
$$

If $h_{i}(P)=h_{i}(Q)=4$ or $h_{i}(P) \neq 4$ and $h_{i}(Q) \neq 4$, then $P_{i 1}^{\prime}$ is obtained from $P_{i 4}$ by the same permutation of columns as $Q_{i 1}^{\prime}$ from $Q_{i 4}$, so $(1)$ is clear. Suppose $h_{i}(P)=4$ 
and $h_{i}(Q) \neq 4$. Then $\left(P_{i 4 k 1}, P_{i 4 k 2}, P_{i 4 k 3}\right)$ is a permutation of $(O, J, J)$ and matrices $Q_{i 4 k 1}, Q_{i 4 k 2}, Q_{i 4 k 3}$ have the same row sum (equal to 1 ). Therefore

$$
\sum_{l=1}^{3} P_{i 1 k l}^{\prime} Q_{i 1 k l}^{\prime T}=\sum_{l=1}^{3} P_{i 4 k l} Q_{i 4 k l}^{T}=2 J
$$

and (1) follows.

It is readily verified that

$$
\sum_{n=0}^{11} \sigma^{n} M=5 J
$$

Thus, the set $\mathcal{M}$, the matrix $M$, and the group $G$ satisfy Lemma 2.1 for $(v, k, \lambda)=$ $(36,15,6)$ with $|G|=12$. Note that the sum of the entries of any row of any matrix $P \in \mathcal{M}$ is equal to 15 .

Let $\bar{M}=J-M$ and $\overline{\mathcal{M}}=\{J-P: P \in \mathcal{M}\}$. Without changing $G$, we obtain that $\overline{\mathcal{M}}, \bar{M}$, and $G$ satisfy Lemma 2.1 for $(v, k, \lambda)=(36,21,12)$. The sum of the entries of any row of any matrix $P \in \overline{\mathcal{M}}$ is equal to 21 .

Note that the described $(36,15,6)$-design and $(36,21,12)$-design are symmetric $\left(4 h^{2}, 2 h^{2}-h, h^{2}-h\right)$-designs with $h=3$ and $h=-3$, respectively.

\section{Using the Kronecker product}

The next lemma will allow us to double the parameter $h$ in a family of symmetric $\left(4 h^{2}, 2 h^{2}-h, h^{2}-h\right)$-designs satisfying Lemma 2.1 .

Lemma 4.1 Let an integer $h \neq 0$, a set $\mathcal{M}$ of matrices of order $4 h^{2}$, and a finite cyclic group $G=\langle\sigma\rangle$ of bijections $\mathcal{M} \rightarrow \mathcal{M}$ satisfy the following conditions:

(i) $\mathcal{M}$ contains the incidence matrix $M$ of a symmetric $\left(4 h^{2}, 2 h^{2}-h, h^{2}-h\right)$-design;

(ii) for any $P, Q \in \mathcal{M},(\sigma P)(\sigma Q)^{T}=P Q^{T}$;

(iii) $\sum_{n=0}^{|G|-1} \sigma^{n} M=\frac{(2 h-1)|G|}{4 h} J$.

(iv) the sum of the entries of any row of any matrix $P \in \mathcal{M}$ is equal to $2 h^{2}-h$.

Then there exists a set $\mathcal{M}_{1}$ of matrices of order $16 h^{2}$ and a cyclic group $G_{1}=\langle\tau\rangle$ of bijections $\mathcal{M}_{1} \rightarrow \mathcal{M}_{1}$ satisfying the following conditions:

(a) $\mathcal{M}_{1}$ contains the incidence matrix $M_{1}$ of a symmetric $\left(16 h^{2}, 8 h^{2}-2 h, 4 h^{2}-2 h\right)$ design;

(b) for any $R, S \in \mathcal{M}_{1},(\tau R)(\tau S)^{T}=R S^{T}$;

(c) $\sum_{n=0}^{\left|G_{1}\right|-1} \tau^{n} M_{1}=\frac{(4 h-1)\left|G_{1}\right|}{8 h} J$

(d) the sum of the entries of any row of any matrix $R \in \mathcal{M}_{1}$ is equal to $8 h^{2}-2 h$;

(e) $\left|G_{1}\right|=2|G|$.

Proof. For any $P \in \mathcal{M}$, define

$$
R_{P}=\left[\begin{array}{cccc}
J-P & P & P & P \\
P & J-P & P & P \\
P & P & J-P & P \\
P & P & P & J-P
\end{array}\right] .
$$


It is well known and readily verified that $M_{1}=R_{M}$ is the incidence matrix of a symmetric $\left(16 h^{2}, 8 h^{2}-2 h, 4 h^{2}-2 h\right)$-design.

Let $\mathcal{M}_{1}=\left\{R_{P}: P \in \mathcal{M}\right\}$. Then $M_{1} \in \mathcal{M}_{1}$, so $\mathcal{M}_{1}$ satisfies (a). Condition (d) is implied by (iv). Any matrix $R \in \mathcal{M}_{1}$ can be divided into eight $4 h^{2}$ by $8 h^{2}$ cells $R_{i j}$, $1 \leq i \leq 4,1 \leq j \leq 2$. Observe that each $R_{i j}$ is of one of the two following types:

(type 1) $R_{i j}=\left[\begin{array}{ll}P & J-P\end{array}\right]$ or $R_{i j}=\left[\begin{array}{ll}J-P & P\end{array}\right], P \in \mathcal{M}$;

(type 2) $R_{i j}=\left[\begin{array}{ll}P & P\end{array}\right], P \in \mathcal{M}$.

Observe also that $R_{i 1}$ and $R_{i 2}$ are not of the same type.

For any $R \in \mathcal{M}_{1}$, denote by $\tau R$ a $(0,1)$-matrix of order $16 h^{2}$ divided into eight $4 h^{2}$ by $8 h^{2}$ cells $\tau R_{i j}, 1 \leq i \leq 4,1 \leq j \leq 2$, where

$$
\tau R_{i 2}=R_{i 1}
$$

and

$$
\tau R_{i 1}= \begin{cases}J-R_{i 2} & \text { if } R_{i 2} \text { is of type } 1 \\
{\left[\begin{array}{ll}
\sigma P & \sigma P
\end{array}\right]} & \text { if } R_{i 2}=\left[\begin{array}{ll}
P & P
\end{array}\right]\end{cases}
$$

In order to verify (b), it suffices to show that, for $i=1,2,3,4,\left(\tau R_{i 1}\right)\left(\tau S_{i 1}\right)^{T}=$ $R_{i 2} S_{i 2}^{T}$.

If $R_{i 2}$ and $S_{i 2}$ are of type (1), then $\left(\tau R_{i 1}\right)\left(\tau S_{i 1}\right)^{T}=\left(J-R_{i 2}\right)\left(J-S_{i 2}\right)^{T}=$ $8 h^{2} J-R_{i 2} J^{T}-J S_{i 2}^{T}+R_{i 2} S_{i 2}^{T}=R_{i 2} S_{i 2}^{T}$ for the row sum of any matrix of type 1 is equal to $4 h^{2}$. If $R_{i 2}=\left[\begin{array}{ll}P & P\end{array}\right]$ and $S_{i 2}=\left[\begin{array}{ll}Q & Q\end{array}\right]$, where $P, Q \in \mathcal{M}$, then $\left(\tau R_{i 1}\right)\left(\tau S_{i 1}\right)^{T}=2(\sigma P)(\sigma Q)^{T}=2 P Q^{T}=R_{i 2} S_{i 2}^{T}$. If $R_{i 2}=\left[\begin{array}{ll}P & P\end{array}\right]$ and $S_{i 2}$ is of type 1 , then $\left(\tau R_{i 1}\right)\left(\tau S_{i 1}\right)^{T}=(\sigma P) J=\left(2 h^{2}-h\right) J=R_{i 2} S_{i 2}^{T}$.

Let $G_{1}$ be the group of bijections $\mathcal{M}_{1} \rightarrow \mathcal{M}_{1}$ generated by $\tau$. Then (e) is satisfied, and we have to verify (c). For $n=1,2, \ldots, 2|G|-1$, let $A_{n}$ be the $(i, j)$-block of the 4 by 4 block-matrix $\tau^{n} M_{1}$. Then there is $P \in \mathcal{M}$ such that the multiset $\left\{A_{n}: 0 \leq n \leq 2|G|-1\right\}$ is the union of $\left\{\sigma^{n} P: 0 \leq n \leq|G|-1\right\}$ and the multiset consisting of $\frac{|G|}{2}$ copies of $P$ and $\frac{|G|}{2}$ copies of $J-P$. Therefore,

$$
\sum_{n=0}^{2|G|-1} A_{n}=\sum_{n=0}^{|G|-1} \sigma^{n} P+\frac{|G|}{2} J=\frac{(2 h-1)|G|}{4 h} J+\frac{|G|}{2} J=\frac{(4 h-1)\left|G_{1}\right|}{8 h} J
$$

The following theorem is now immediate by induction.

Theorem 4.2 Let an integer $h \neq 0$, a set $\mathcal{M}$ of matrices of order $4 h^{2}$, and a finite cyclic group $G$ of bijections $\mathcal{M} \rightarrow \mathcal{M}$ satisfy conditions (i)-(iv) of Lemma 4.1. Then, for any positive integer $d$, there exists a non-empty set $\mathcal{M}_{d}$ of matrices of order $4^{d+1} h^{2}$ and a cyclic group $G_{d}$ of bijections $\mathcal{M}_{d} \rightarrow \mathcal{M}_{d}$ satisfying the following conditions:

(a) $\mathcal{M}_{d}$ contains the incidence matrix $M_{d}$ of a symmetric design with parameters

$$
\left(4^{d+1} h^{2}, 2^{2 d+1} h^{2}-2^{d} h, 2^{2 d} h^{2}-2^{d} h\right) ;
$$

(b) for any $P, Q \in \mathcal{M}_{d}$ and $\tau \in G_{d},(\tau P)(\tau Q)^{T}=P Q^{T}$; 
(c) $\sum_{\tau \in G_{d}} \tau M_{d}=\frac{\left(2^{d+1} h-1\right)\left|G_{d}\right|}{2^{d+2} h} J$; $2^{d} h$;

(d) the sum of the entries of any row of any matrix $R \in \mathcal{M}_{d}$ is equal to $2^{2 d+1} h^{2}-$

(e) $\left|G_{d}\right|=2^{d}|G|$.

We combine Theorem 4.2 and Lemma 2.1 and obtain the main result of this paper.

Theorem 4.3 If $h= \pm 3 \cdot 2^{d}$, where $d$ is a positive integer and $|2 h-1|$ is a prime power, then, for any positive integer $m$, there exists a symmetric $\left(\frac{h\left((2 h-1)^{2 m}-1\right)}{h-1}, h(2 h-\right.$ $\left.1)^{2 m-1}, h(h-1)(2 h-1)^{2 m-2}\right)$-design.

Proof. We start with the set $\mathcal{M}$ or $\overline{\mathcal{M}}$ described in Section 3 and apply Theorem 4.2 to this set to obtain the set of matrices $\mathcal{M}_{d}$ or $\overline{\mathcal{M}}_{d}$ and the group $G_{d}$. Then we apply Lemma 2.1. Properties (ii) and (iii) required in Lemma 2.1 are implied by (b) and (c) of Theorem 4.2. The parameter $q$ of Lemma 2.1 is equal to $\left(2 h_{d}-1\right)^{2}$, where $h_{d}= \pm 3 \cdot 2^{d}$, so $q$ is a prime power. Since $|G|=12$, we have $\left|G_{d}\right|=3 \cdot 2^{d+2}=4\left|h_{d}\right|$, so $\left|G_{d}\right|$ divides $q-1$.

Remark 4.4 These parameters are new, except $m=1$ (Menon designs).

\section{References}

[1] T. Beth, D. Jungnickel, and H. Lenz, Design Theory, B.I. Wissenschaftverlag, Mannheim, 1985, Cambridge Univ. Press, Cambridge, UK, 1986.

[2] A. E. Brouwer, An infinite series of symmetric designs, Math. Centrum Amsterdam Report, ZW $136 / 80(1983)$.

[3] The CRC Handbook of Combinatorial Designs, eds. C. J. Colbourn and J. H. Dinitz, CRC Press, 1996.

[4] J. D. Fanning, A family of symmetric designs, Discrete Mathematics 146 (1995), pp. 307-312.

[5] Y.J. Ionin, Symmetric subdesigns of symmetric designs, Journal of Combinatorial Mathematics and Combinatorial Computing (to appear).

[6] Y.J. Ionin, A technique for constructing symmetric designs, Designs, Codes and Cryptography (to appear).

[7] D. Jungnickel, Difference sets, in: Contemporary Design Theory: A Collection of Surveys (J.H. Dinitz, D.R. Stinson; eds.), John Wiley \& Sons, New York, 1992, 241-324.

[8] D.P. Rajkundlia, Some techniques for constructing infinite families of BIBDs, Discrete Math. 44 (1983), 61-96.

[9] J. Seberry and M. Yamada, Hadamard matrices, sequences, and block designs, in Contemporary Design Theory, eds. J.H. Dinitz and D.R. Stinson, John Wiley \& Sons, 1992, 431-560. 